\title{
ANÁLISES LABORATORIAIS FISCAIS EM PRODUTOS LACTÉOS DE AGROINDÚSTRIAS FAMILIARES DO ESTADO DO ESPÍRITO SANTO COM ENFÂSE NA IDENTIFICAÇÃO DE Listeria monocytogenes
}

\author{
FISCAL LABORATORY ANALYSIS IN DAIRY PRODUCTS FROM FAMILY \\ AGRO-INDUSTRIES OF THE STATE OF ESPÍRITO SANTO WITH \\ EMPHASIS ON IDENTIFICATION OF Listeria monocytogenes
}

\author{
${ }^{1}$ Jamili Maria Suhet Mussi \\ ${ }^{2 *}$ Gabriel Domingos Carvalho \\ ${ }^{3}$ Flávia Regina Spago
}

\begin{abstract}
${ }^{1}$ Instituto Federal do Espírito Santo - Ifes Campus Piúma. E-mail: jamilisuhet@gmail.com ${ }^{2}$ Instituto Federal do Espírito Santo - Ifes Campus Piúma. E-mail: gabriel.carvalho@ifes.edu.br ${ }^{3}$ Instituto Federal do Espírito Santo - Ifes Campus Piúma. E-mail: flavia.goncalves@ifes.edu.br

*Autor de correspondência
\end{abstract}

Artigo submetido em 15/12/2020, aceito em 25/06/2021 e publicado em 26/07/2021.

Resumo: No Espírito Santo a produção de lacticínios é uma atividade muito importante para as agroindústrias familiares, havendo uma forte relação cultural e manutenção dessas famílias no campo. Dependendo das condições higiênicas durante o processo de ordenha do leite e do preparo dos produtos, bem como aspectos relacionados à sanidade do rebanho, estes produtos podem ser contaminados por diferentes micro-organismos patogênicos, podendo ocasionar perdas econômicas para indústria e sérios problemas de saúde pública. Dessa forma, a realização de análises laboratoriais, especialmente microbiológicas, deve ser periódica, visando garantir a inocuidade do produto e a segurança dos consumidores. Especificamente para queijos, a presença da bactéria Listeria monocytogenes está entre os parâmetros avaliados devido sua relevância para saúde pública. Este estudo realizou um levantamento retrospectivo das análises laboratoriais fiscais do ano de 2019, com enfâse na identificação de L. monocytogenes, em agroindústrias familiares produtoras de lacticínios registradas em Serviços de Inspeção Municipais com equivalência reconhecida e adesão ao Sistema Unificado Estadual de Sanidade Agroindustrial Familiar de Pequeno Porte - Susaf/ES. Todos os municípios que possuem agroindústrias incluídas no Susaf/ES realizaram análises fiscais em 2019, sendo que a maioria dos estabelecimentos amostrou um único produto em uma análise anual, na qual foi analisada uma variedade de produtos distintos, com predomínio de queijos. A maioria das análises foram realizadas em laboratório credenciado junto ao Idaf. Os resultados das análises foram negativos para presença de L. monocytogenes, correspondendo com a legislação, reforçando a importância do registro e do acompanhamento do estabelecimento pelos serviços de inspeção enquanto órgão fiscalizador.

Palavras-chave: inspeção; listeriose; queijo; segurança alimentar.

Abstract: In Espírito Santo State, the dairy production is an activity of great importance for family agro-industries with a strong cultural relationship with the maintenance of families in the countryside. However, depending on the hygienic conditions during the milking process and the preparation of the dairy products and also on the health of the herd, these products may be contaminated by different pathogenic microorganisms, which may cause economic losses for industry and serious problems for 
public health. Thus, laboratory tests, especially the microbiological tests, must be carried out periodically, aiming to guarantee the safety of the product and the safety for the consumers. Specifically for cheeses, the presence of the bacterium Listeria monocytogenes is among the parameters evaluated in dairy products, due to its relevance to public health. This study carried out a retrospective survey of the fiscal laboratory analyzes, during 2019, with an emphasis on the identification of L. monocytogenes, in family agro-industries registered in the Municipal Inspection Services - SIM with equivalence to the Susaf/ES. All municipalities that have agro-industries equivalent to Susaf/ES underwent fiscal analyzes in 2019, with the majority of establishments sampling a single product in an annual analysis, been sampling a variety of distinct dairy products, with a predominance of cheeses. Most analyzes were performed in a laboratory accredited by Idaf. The results of analyzes were negative for the presence of $L$. monocytogenes, corresponding to the legislation, reinforcing the importance of the registration and monitoring of the establishment by the inspection services.

Keywords: cheese; food security; inspection; listeriosis.

\section{INTRODUÇÃO}

O estado do Espírito Santo possui 1.651 agroindústrias que se dedicam à fabricação de diferentes produtos, incluindo doces, bebidas, conservas, produtos lácteos, embutidos, carnes defumadas e socol, tendo distribuição em todo o estado, especialmente na região metropolitana. Destas, aproximadamente $30 \%$ são estabelecimentos que processam produtos de origem animal, com destaque para produção de queijos, representando $64,2 \%$ do total dos produtos elaborados, e ainda destaque para produção de derivados de carne suína e outros derivados lácteos segundo o Instituto Capixaba de Pesquisa e Extensão Rural - Incaper (VINHA; DIAS, 2019).

Medidas de controle higiênicosanitários devem ser realizadas durante a elaboração de produtos de origem animal, para assegurar a segurança alimentar. As agroindústrias devem ser inspecionadas e fiscalizadas por órgãos competentes, federais, estaduais ou municipais (BRASIL, 2017). Os estabelecimentos devem adotar medidas para prevenir e eliminar a contaminação por microorganismos patogênicos e realizar análises laboratoriais para identificação destes agentes (BRASIL, 2009; ESPÍRITO SANTO, 2014).
No Brasil, diversos órgãos e legislações estão relacionados com a inspeção e fiscalização de alimentos, como a Agência Nacional de Vigilância Sanitária (Anvisa) e o Ministério da Agricultura Pecuária e Abastecimento (MAPA), e preconizam a necessidade de realização de análises microbiológicas para garantir que o alimento possa ser considerado seguro para o consumo humano (BRASIL, 1996; BRASIL, 2017; BRASIL, 2019a; BRASIL, 2019b).

Os parâmetros microbiológicos que devem ser avaliados irão variar com o tipo de derivados lácteos amostrados e com legislação adotada como base de avaliação, podendo comtemplar análises para Enterobacteriaceae/mL ou g, Salmonella/ $25 \mathrm{~g}$ ou $\mathrm{mL}$, Escherichia coli/g, Coliformes $\left(\begin{array}{llll}30 & \text { e } & 45 & { }^{\circ} \mathrm{C}\end{array}\right) / \mathrm{g}$ ou $\mathrm{mL}$, Estafilococos coagulase positiva/g, Enterotoxinas estafilocócicas (ng/g), Bolores e leveduras/g, Aeróbios mesófilos/g, Bacillus cereus presuntivo/g e L. monocytogenes/25g ou mL (BRASIL, 2019a; BRASIL, 2019b).

Com a ampliação da fabricação de produtos de origem animal nos municípios capixabas, alguns produtores familiares visam comercializar seus produtos além das fronteiras dos municípios. Pensando nisso, o Governo do Estado, através do Instituto de Defesa Agroflorestal do Espírito Santo (Idaf), instituiu o Sistema 
Unificado Estadual de Sanidade Agroindustrial Familiar de Pequeno Porte (Susaf/ES), que promove a equivalência entre os serviços de inspeção municipais e o Estadual. Este sistema objetiva padronizar os procedimentos de registro, inspeção e fiscalização entre os órgãos, permitindo a ampliação do comércio das agroindústrias familiares. Além disso, há um ganho em saúde pública, pois os municípios com a equivalência devem seguir os requisitos do órgão estadual, entre eles a apresentação de cronograma de execução de análises laboratoriais pelos empreendimentos, de forma a ofertar produtos seguros para os consumidores, evitando a transmissão de agentes patogênicos pelo alimento (IDAF, 2020a), incluindo Listeria monocytogenes.

Neste contexto, objetivou-se com este trabalho realizar um levantamento retrospectivo da realização de análises laboratoriais fiscais no ano de 2019, com enfâse na identificação de Listeria monocytogenes, em agroindústrias familiares de produtos lácteos, registradas em Serviços de Inspeção Municipais, em municípios com equivalência reconhecida e adesão ao Susaf/ES, em atendimento a legislaçao vigente.

\section{REFERENCIAL TEÓRICO}

\subsection{Listeria monocytogenes e a indústria de alimentos}

O gênero Listeria é composto por várias espécies, porém cinco são rotineiramente isoladas de alimentos, como L. monocytogenes, L. inocua, L. ivanovii, L. seeligeri e $L$. welshimeri. Destas, a espécie de maior importância em saúde pública, com ocorrência de surtos de origem alimentar, é L. monocytogenes (THOUVENOT et al., 2018).

L. monocytogenes é um bastonete Gram positivo, anaeróbio facultativo, catalase positivo, que cresce em ampla faixa de temperatura de 1 a $45^{\circ} \mathrm{C}$ e em $\mathrm{pH}$ 4,5 a 9,6. O patógeno é capaz de sobreviver na presença de elevadas concentrações salinas, de até 15\%, e atividade de água de 0,94 ou menos (VALLIM et al., 2015).

Em decorrência da gravidade que a listeriose causa em seres humanos, especialmente em imunocomprometidos (náuseas, dores de cabeça e musculares, perda de equilíbrio e convulsões) e gestantes (abortos e natimortos) e da taxa de letalidade da doença, em torno de 20$30 \%$, a ingestão de leite e derivados lácteos contaminados com L. monocytogenes é fator preocupante com relação a saúde pública (CHOI et al., 2018).

Além de causar doenças em seres humanos, L. monocytogenes provoca perdas econômicas significativas na indústria de alimentos, visto que a bactéria é de difícil eliminação nas instalações industriais, podendo levar a contaminação de diferentes lotes de produtos (CAMARGO et al., 2017). A indústria que produzir alimento contaminado poderá estar sujeita a diferentes penalidades pelos órgãos de fiscalização, como destruição do produto, comprovação da adoção de medidas para eliminação da bactéria por realização de exames laboratoriais, entre outras (BRASIL, 2009; ESPÍRITO SANTO, 2014).

Diversas podem ser as fontes de contaminação dos produtos lácteos por $L$. monocytogenes, como o leite obtido de animais infectados ou o uso de água contaminada, bem como falhas de higienização dos tetos e equipamentos durante o processo de ordenha (PAPIĆ et al., 2019). Além disso, na indústria também há a possibilidade de contaminação, principalmente em virtude do manuseio de produtos com higienização inadequada, limpeza ineficiente de equipamentos e instalações, falhas nas etapas de embalagem, distribuição e armazenamento do produto. O patógeno é capaz de sobreviver em diversos materiais, incluindo aqueles utilizados para embalagem dos produtos acabados e de 
multiplicar em baixas temperaturas, formando biofilmes, devendo os manipuladores atentarem para que não ocorra contaminação cruzada entre as etapas de processamento (DI CICCIO et al., 2020).

A viabilidade de $L$. monocytogenes nos produtos lácteos é dependente dos parâmetros intrínsecos e extrínsecos do alimento. Fatores como atividade de água, $\mathrm{pH}$, contaminação inicial, presença de outros micro-organismos competidores, condições de processamento, formas e temperatura de armazenamento e de maturação podem influenciar na sobrevivência de L. monocytogenes (WEMMENHOVE et al., 2016; GÉRARD et al., 2018; LEE et al., 2020).

Os queijos se destacam entre os derivados lácteos em relação a contaminação por L. monocytogenes. A sobrevivência do micro-organismo é variável nos diferentes tipos, sendo que em queijos mais frescos, com maior umidade e menor acidificação há maior possibilidade da presença do patógeno do que em queijos maturados por longos períodos, onde a atividade de água é reduzida (GÉRARD et al., 2018). Além disso, em queijos produzidos com leite cru, o risco pode ser mais elevado, quando comparado àqueles que passaram pela etapa de pasteurização do leite (GANZ et al., 2020). Vale ressaltar que mesmo em queijos maturados por longo período o patógeno pode permanecer viável. Em um estudo com queijo italiano, contaminado experimentalmente, a redução significativa do patógeno só foi constatada após 60 dias de maturação, porém foi possível a detecção da bactéria até o final do estudo, aos 153 dias, em que o pH foi próximo a 5 e atividade de água de 0,91 (GIACOMETTI et al., 2020).

Além do queijo, outros derivados lácteos também são passíveis de contaminação pelo micro-organismo. Em iogurte, Listeria pode sobreviver ao processo de fermentação e estar viável após 30 dias de fabricação, com pH em torno de 4,0. Em manteiga, foi demonstrada a sobrevivência de $L$. monocytogenes durante o armazenamento do produto congelado $\left(-18^{\circ} \mathrm{C}\right)$ por 70 dias (BARANCELLI et al., 2011).

Apesar de ainda não ter sido comprovada a ocorrência de casos de listeriose associado ao consumo de leite e produtos lácteos no Brasil, $L$. monocytogenes já foi isolada de leite cru, leite pasteurizado e outros produtos lácteos, inclusive queijos de diferentes variedades. Acredita-se que o principal fator responsável pela ausência de casos humanos no país, por ingestão destes produtos, seja pela listeriose ser subdiagnosticada e subnotificada (OLIVEIRA et al., 2019).

\subsection{Exigências legais para análises de Listeria monocytogenes em produtos lácteos}

No Brasil é estabelecido como padrão para pesquisa de $L$. monocytogenes, em leite e derivados, tanto em alimentos coletados nas indústrias processadoras, pelo MAPA, quanto para alimentos coletados no comércio, pela Anvisa, a ausência da bactéria em 25g ou mL do produto (BRASIL, 1996; BRASIL, 2019a; BRASIL, 2019b).

A Anvisa estabelece os critérios e padrões microbiológicos aceitáveis para alimentos prontos para o consumo, sendo que a realização da análise para $L$. monocytogenes só não é exigida em situações definidas, como por exemplo em alimentos com vida útil menor que 5 dias, $\mathrm{pH}$ igual ou inferior a 4,4, atividade de água inferior ou igual a 0,92 , combinação de $\mathrm{pH}$ menor ou igual a 5,0 e atividade de água menor ou igual a 0,94 , realização de tratamento térmico que elimine o microorganismo e cuja recontaminação após este tratamento não seja possível (BRASIL, 2019a).

Já o MAPA define os parâmetros laoratoriais a serem analisados para os 
diversos produtos, sendo que para os derivados lácteos a pesquisa de $L$. monocytogenes é definida para diversas variedades de queijos maturados e não maturados, porém não é exigida nos demais produtos, como requeijão, doce de leite, manteiga e iogurte (BRASIL, 1996, BRASIL, 2019b).

O MAPA também institui procedimentos específicos para o controle de $L$. monocytogenes em produtos de origem animal, visando monitorar e assegurar a inocuidade destes alimentos, os quais incluem a realização de análises laboratoriais e a inspeção dos processos de produção. Na ocorrência de resultados positivos para L. monocytogenes, deverão ser revisados os registros dos produtos, os programas de autocontrole, avaliada a higiene das instalações e equipamentos, hábitos de manipuladores e condições da matéria-prima. Além disso, o estabelecimento deverá apresentar medidas preventivas, corretivas e de monitoramento que serão aplicadas para resolução da contaminação pelo patógeno, além de realizar análises laboratoriais para liberação dos produtos. No entanto, na constatação de que as ações para resolver a contaminação não foram aplicadas ou efetivas, medidas como a apreensão dos produtos e exigência de realização de novas análises laboratoriais para pesquisa de $L$. monocytogenes poderão ser aplicadas (BRASIL, 2009).

O Idaf também institui a necessidade de realização de análises laboratoriais de produtos de origem animal. Para o Serviço de inspeção estadual estão normatizados os procedimentos a que serão submetidas às empresas com não conformidades nas análises laboratóriais, denominado de Regime Especial de Fiscalização (REF), que envolve medidas como a suspensão da produção, interdição parcial ou total do estabelecimento, apreensão e inutilização dos produtos apreendidos, necessidade de acompanhamento fiscal para a fabricação, recolhimento de produtos já introduzidos nos mercados de consumo, entre outras medidas corretivas. O estabelecimento só será liberado do REF após realização de análises laboratoriais que comprovem a resolução da inconformidade (ESPÍRITO SANTO, 2014).

Os municípios também possuem nas legislações de implantação dos Serviços de Inspeção Municipais a exigência de análises laboratoriais para os produtos elaborados em estabelecimentos registrados, sendo que para manutenção da equivalência com o Susaf/ES, estas análises devem ser realizadas de acordo com um cronograma pré-estabelecido e os registros de realização, bem como as providências adotadas em relação às análises fora do padrão, devem ser arquivados de forma auditável (ESPÍRITO SANTO, 2018).

\section{MATERIAIS E MÉTODOS}

Realizou-se um levantamento retrospectivo das análises laboratoriais para a identificação de $L$. monocytogenes, realizadas em 2019, em agroindústrias beneficidoras de produtos lácteos, registradas em Serviços de Inspeção Municipais e com equivalência reconhecida e adesão ao Sistema Unificado Estadual de Sanidade Agroindustrial Familiar de Pequeno Porte - Susaf/ES.

Os municípios capixabas participantes do Susaf/ES, em 2019, foram: Venda Nova do Imigrante, Domingos Martins, Santa Maria de Jetibá, Castelo, Guarapari, Linhares, Cariacica e Cachoeiro de Itapemirim (IDAF, 2020a). O número de agroindústrias de produtos lácteos, por município, incluídas no Susaf/ES estão representadas na Tabela 1.

Foi solicitado aos Serviços de Inspeção Municipais, das cidades participantes do Susaf/ES, os resultados das análises microbiológicas para $L$. monocytogenes e análise físico-química de umidade, realizadas entre janeiro e 
dezembro de 2019, nas agroindústrias incluídas no sistema.

Os dados obtidos a partir dos resultados das análises laboratoriais realizadas foram tabulados e procedeu-se a análise descritiva das mesmas, tendo como base os valores de referência das legislações brasileiras - MAPA e Anvisa (BRASIL, 1996; BRASIL, 2019a, 2019b).

\section{RESULTADOS E DISCUSSÃO}

Dos municípios participantes do Susaf/ES, a maior parte (6) possuiam agroindústrias produtoras de derivados lácteos incluídas no Susaf/ES (IDAF, 2020a), sendo que todas $(\mathrm{n}=11)$ realizaram análises laboratoriais microbiológicas no ano de 2019. Os municípios de Castelo e Guarapari não possuiam estabelecimento da categoria incluídos no Susaf/ES até o final do ano de 2019 (Tabela 1).

Tabela 1: Municípios capixabas com agroindústrias produtoras de derivados lácteos registradas no SIM e incluídas no Susaf/ES.

\begin{tabular}{|c|c|c|}
\hline Município & $\begin{array}{l}\text { Número de } \\
\text { agroindústrias } \\
\text { com SIM }\end{array}$ & $\begin{array}{c}\text { Número de } \\
\text { agroindústrias } \\
\text { com Susaf/ES }\end{array}$ \\
\hline $\begin{array}{l}\text { Cachoeiro de } \\
\text { Itapemirim }\end{array}$ & 4 & 1 \\
\hline Cariacica & 2 & 2 \\
\hline Castelo & 7 & 0 \\
\hline $\begin{array}{l}\text { Domingos } \\
\text { Martins }\end{array}$ & 6 & 4 \\
\hline Guarapari & 5 & 0 \\
\hline Linhares & 3 & 1 \\
\hline $\begin{array}{l}\text { Santa Maria de } \\
\text { Jetibá }\end{array}$ & 1 & 1 \\
\hline $\begin{array}{l}\text { Venda Nova do } \\
\text { Imigrante }\end{array}$ & 9 & 2 \\
\hline Total & 37 & 11 \\
\hline
\end{tabular}

Fonte: Idaf (2020a).

A frequência, quantidade de amostras por estabelecimento e tipo de produto analisado foi diferente em cada estabelecimento (Tabela 2). Dentre os tipos de produtos amostrados, pode-se observar que os queijos do tipo Minas frescal e Minas frescal temperado foram classificados como de muito alta umidade (igual ou maior a 55\%), os queijos Minas, Minas padrão e ricota foram classificados com alta umidade (entre $46 \%$ e $54,9 \%$ ), já o queijo coalho e o requeijão em barra apresentaram umidade média (de 36 a $45,9 \%$ ) e, apenas o queijo muçarela apresentou baixa umidade (13\%), conforme a legislação (BRASIL, 1996).

Apenas dois estabelecimentos não contemplaram a realização da análise de pesquisa para $L$. monocytogenes em produtos amostrados (doce de leite e iogurte), pois este parâmetro não está previsto no escopo de análise dos produtos (BRASIL, 2019b). Para o doce de leite o estabelecimento só produz este tipo de produto, já o estabelecimento produtor do iogurte realizou a pesquisa do patógeno para o produto queijo Minas frescal (Tabela 2). Vale ressaltar que, apesar da legislação brasileira não prever a realização de análises laboratoriais para detecção de Listeria monocytogenes em todos os tipos de produtos lácteos, não é possível assegurar a ausência do patógeno nos mesmos, tanto pela possibilidade de contaminação pós processamento, quanto pela descrição na literatura da sobrevivência do patógeno em derivados lácteos diversos, incluindo no doce de leite, manteiga e iogurte (HENTGES et al., 2010; BARANCELLI et al., 2011; SZCZAWIŃSKI et al., 2016).

Todos os tipos de queijos e no requeijão das agroindústrias incluídas no Susaf/ES, que realizaram análises laboratoriais microbiológicas no ano de 2019, apresentaram resultados de ausência de $L$. monocytogenes em 25 gramas do produto (Tabela 3), estando estes resultados em conformidade com as legislações brasileiras tanto do MAPA como da Anvisa (BRASIL, 1996; BRASIL, 2019a). 
O controle de patógenos em produtos lácteos, principalmente em queijos, está relacionado com a umidade do produto, com variação da atividade de água disponível para o crescimento microbiano, com alterações do $\mathrm{pH}$ e da temperatura de armazenamento (THOMAS; TIWARI; MISHRA, 2019; DONNELLY, 2004). Em queijos mais frescos estes parâmetros são mais elevados, o que facilita o crescimento e multiplicação dos micro-organismos. Além disso, queijos mais frescos, como o Minas frescal, são submetidos à manipulação manual mais acentuada durante fabricação, facilitando assim a contaminação, sobrevivência e multiplicação de bactérias patogênicas, como L. monocytogenes (OLIVEIRA et al., 2019). A redução da sobrevivência de $L$. monocytogenes em queijos foi observada com a redução da umidade ao longo da maturação (CALLON et al., 2011).

Tabela 2: Caracterização da amostragem e das análises laboratoriais realizadas no ano de 2019 pelas agroindústrias de derivados lácteos registrados no SIM e incluídas no Susaf/ES.

\begin{tabular}{|c|c|c|c|c|c|}
\hline Agroindústria & $\begin{array}{c}\text { Frequência de } \\
\text { análises } \\
\text { realizadas }\end{array}$ & $\begin{array}{l}\text { Tipos de produtos } \\
\text { processados na } \\
\text { agroindústria }\end{array}$ & $\begin{array}{l}\text { Número de } \\
\text { produtos } \\
\text { amostrados }\end{array}$ & $\begin{array}{l}\text { Tipo de produto } \\
\text { amostrado }\end{array}$ & $\begin{array}{l}\text { Umidade do } \\
\text { produto } \\
\text { amostrado } \\
\end{array}$ \\
\hline \multirow[t]{2}{*}{ A } & 1 & Queijos & 2 & Queijo coalho & $45,1 \%$ \\
\hline & & Iogurtes & & Queijo frescal temperado & 57,1 \\
\hline \multirow[t]{5}{*}{ B } & 1 & Queijos & 4 & Queijo Minas padrão & $52,2 \%$ \\
\hline & & Requeijão & & Ricota fresca light & não realizado \\
\hline & & Ricota & & Queijo Minas frescal & $55 \%$ \\
\hline & & Manteiga & & Requeijão em barra & \\
\hline & & Iogurtes & & & $38 \%$ \\
\hline C & 1 & Doce de leite & 1 & Doce de leite & não realizado* \\
\hline D & 1 & Queijo Minas & 1 & Queijo Minas & $51 \%$ \\
\hline $\mathbf{E}$ & 1 & Queijo Minas & 1 & Queijo Minas & $49 \%$ \\
\hline $\mathbf{F}$ & 1 & Queijo Minas & 1 & Queijo Minas & $52 \%$ \\
\hline $\mathbf{G}$ & 1 & Queijo Minas frescal & 1 & Queijo Minas frescal & $61,48 \%$ \\
\hline \multirow[t]{4}{*}{$\mathbf{H}$} & 1 & Queijos & 1 & & \\
\hline & & Iogurtes & & Queijo Minas & $49 \%$ \\
\hline & & Doce de leite & & & \\
\hline & & Puína & & & \\
\hline $\mathbf{I}$ & 1 & Queijo & 1 & Queijo Minas padrão & $47,5 \%$ \\
\hline \multirow[t]{5}{*}{$\mathbf{J}$} & 2 & Queijos & 3 & Queijo muçarela de búfala & $13 \%$ \\
\hline & & Requeijão & & Ricota fresca & $48,30 \%$ \\
\hline & & Puína & & Queijo Minas & $43,70 \%$ \\
\hline & & Manteiga & & & \\
\hline & & Doce de leite & & & \\
\hline \multirow[t]{3}{*}{$\mathbf{K}$} & 1 & Queijo & 2 & Queijo Minas frescal & $56,3 \%$ \\
\hline & & Iogurte & & Iogurte & não realizado* \\
\hline & & Ricota & & & \\
\hline
\end{tabular}

\footnotetext{
*Análise de umidade para doce de leite e iogurte por não fazer parte do escopo analítico (BRASIL, 2019b).
} 
Tabela 3: Caracterização das análises laboratoriais para Listeria monocytogenes realizadas no ano de 2019 pelas agroindústrias registradas no SIM e incluídas no Susaf/ES.

\begin{tabular}{ccccc}
\hline Agroindústria & $\begin{array}{c}\text { Resultado da } \\
\text { análise para } \mathbf{L} . \\
\text { monocytognes }\end{array}$ & $\begin{array}{c}\text { Laboratório de } \\
\text { realização da } \\
\text { análise }\end{array}$ & $\begin{array}{c}\text { Norma de qualidade } \\
\text { implantada no } \\
\text { laboratório }\end{array}$ & $\begin{array}{c}\text { Tipo de credenciamento } \\
\text { do laboratório }\end{array}$ \\
\hline A & negativo* & Laboratório 1 & ISO $^{1} 17025$ & RELAGRO \\
B & negativo & Laboratório 1 & ISO 17025 & RELAGRO \\
C & negativo & Laboratório 1 & ISO 17025 & RELAGRO \\
D & negativo & Laboratório 1 & ISO 17025 & RELAGRO \\
E & negativo & Laboratório 1 & ISO 17025 & RELAGRO \\
F & negativo & Laboratório 1 & ISO 17025 & RELAGRO \\
G & negativo & Laboratório 2 & ISO 9001 & Não possui credenciamento \\
H & negativo & Laboratório 2 & ISO 9001 & Não possui credenciamento \\
I & negativo & Laboratório 1 & ISO 17025 & RELAGRO \\
J & negativo & Laboratório 1 & ISO 17025 & RELAGRO \\
K & negativo & Laboratório 3 & ISO 17025 & RELAGRO \\
\hline
\end{tabular}

*negativo = ausência em 25 gramas do produto; ${ }^{1}$ ISO = International Organization for Standardization (Organização Internacional de Padronização); ${ }^{2}$ RELAGRO = Rede Estadual de Laboratórios Agropecuários.

Mediante os resultados das análises dos produtos amostrados, tanto fresco como maturados, com ausência de $L$. monocytogenes em todos os estabelecimentos, pode-se supor que os procedimentos de boas práticas de fabricação e higienização estão sendo realizados de forma adequada.

A amostragem de queijos mais frescos, com alta umidade, poderia contribuir para detecção de $L$. monocytogenes, se a origem da contaminação do produto pelo patógeno fosse o leite. A alimentação de animais com silagem contaminada pode levar a contaminação do leite e, consequentemente, do produto final (PAPIĆ et al., 2019), por isso é fundamental que os estabelecimentos realizem o registro dos fornecedores de matérias-primas, contribuindo para a rastreabilidade na cadeia produtiva (BRASIL, 2018a).

Todos os queijos amostrados para as análises realizadas foram produzidos com leite pasteurizado. A destruição de $L$. monocytogenes no processo de pasteurização está relacionada à carga microbiana no leite. $\mathrm{Na}$ concentração de $10^{2} \mathrm{UFC} / \mathrm{mL}, \quad$ L. monocytogenes é destruída na pasteurização, mas na concentração de $10^{7} \mathrm{UFC} / \mathrm{mL}$, o microorganismo pode sobreviver ao processo de pasteurização (KASALICA et al., 2011). No entanto, é pouco provável a contaminação do leite com concentração bacteriana superior a $280 \mathrm{UFC} / \mathrm{mL}$ (HUNT et al., 2012), sendo esta uma das justificativas possíveis para a ausência do patógeno nas análises que amostraram queijos mais frescos.

Há também a possibilidade de sobrevivência de $L$. monocytogenes em queijos maturados, de acordo com a variedade e umidade, como os queijos tipo Minas, Minas padrão, muçarela e coalho que são maturados em câmara de maturação por períodos e temperaturas variáveis. A sobrevivência de $L$. monocytogenes ao processo de maturação está relacionada com fatores intrínsecos e extrínsecos do processo e do produto, como por exemplo temperatura, umidade, $\mathrm{pH}$, microbiota competidora, concentração 
salina, sendo que o patógeno pode sobreviver em queijos maturados por longos períodos, superior a 60 dias (D'AMICO; DRUART DONNELLY, 2008; WEMMENHOVE et al., 2016; GIACOMETTI et al., 2020).

O processo de maturação em câmaras refrigeradas pode propiciar a contaminação dos queijos póspasteurização. L. monocytogenes possui a capacidade de sobreviver e se multiplicar em temperaturas de refrigeração e de formar biofilmes, 0 que facilita 0 desenvolvimento e persistência do patógeno em diferentes pontos das instalações das fábricas de produtos lácteos (CAMARGO et al., 2017).

De acordo com os resultados e com informações dos serviços de inspeção municipais, as análises laboratoriais não foram realizadas por lotes, sendo realizada a amostragem de um único produto por estabelecimento, definido pelo fiscal no ato da coleta. Desta forma, a coleta de pequeno número de amostras, com ausência de diferentes lotes e de diferentes produtos, pode não ser suficiente para afirmar a ausência de contaminação por $L$. monocytogenes nos estabelecimentos avaliados. Para maior avaliação da situação sanitária do estabelecimento, e consequetentemente maior segurança alimentar, o ideal seria que fosse possível a realização de amostragem por lotes de produtos, com maior variedade, como queijos frescos e maturados e demais produtos lácteos, de um mesmo estabelecimento, aumentando a possibilidade de detecção do patógeno.

Vale ressaltar, que nas câmaras de maturação é indicado realizar a identificação dos lotes de queijos produzidos, facilitando a amostragem e a adoção de medidas corretivas em casos de análises laboratoriais não conformes, tendo como base a crescente demanda de rastreabilidade dos produtos lácteos (PANT; PRAKASH; FAROOQUIE, 2015). Outro ponto importante é a execução rotineira de um programa de higienização das instalações, contemplando, além da força mecânica na limpeza, o uso de produtos como detergentes e sanitizantes em concentrações adequadas, capazes de remover a matéria orgânica e eliminar $L$. monocytogenes nos ambientes de produção, com prevenção da formação de biofilmes (SKOWRON et al., 2018). A eficiência destes procedimentos poderá ser verificada tanto pela análise final dos produtos, como pelas análises amostrais das superfícies do ambiente produtivo, com uso de suabes, por exemplo (COSTA DIAS, 2012).

Nos queijos coalho, muçarela e o requeijão, que passam por etapas de acidificação, com produção de diversos ácidos orgânicos e redução do pH, e por processo de filagem e cozimento em altas temperaturas, a redução da concentração ou inativação de eventuais patógenos como L. monocytogenes podem ocorrer, porém estes processamentos não eliminam a possibilidade de contaminação após o processo de produção (TIRLONI et al., 2019; GÉRARD et al., 2018). Desta forma, a amostragem de queijos mais ácidos ou cozidos podem limitar as chances de detecção de patógenos nas análises laboratoriais.

A maioria dos municípios realizou, no ano de 2019, apenas uma análise laboratorial, com amostragem de um único produto por estabelecimento, sendo adotada a amostragem indicativa (Tabela 2) (IDAF, 2020b).

Os municípios com Susaf/ES necessitam apresentar anualmente ao órgão fiscalizador os programas de trabalho que contemplem o cronograma da realização das análises laboratoriais, e manter os resultados destas de forma auditável (ESPÍRITO SANTO, 2018). Porém, o Idaf ainda não definiu em legislação específica qual a periodicidade da realização das análises e qual a amostragem que deverá ser realizada nos estabelecimentos com Susaf/ES, sendo que cada município possui 
autonomia de definir em seus programas de trabalho como as análises laboratoriais serão realizadas. Vale considerar que o Idaf estabeleceu para o Serviço de Inspeção Agroindustrial de Pequeno Porte (Siapp), semelhante ao Susaf/ES, frequência quadrimestral para a realização de análises laboratoriais fiscais microbiológicas em estabelecimento de leite e derivados com registro definitivo, com coleta de um produto por categoria, priorizando produtos de maior risco (IDAF, 2020c).

Os Serviços de inspeção municipais pesquisados consideram que o alto custo das análises laboratoriais é fator relevante para definição do cronograma de realização das análises laboratoriais nos programas de trabalho, limitando coleta de maior variedade de produtos e maior frequência de realização das análises fiscais em estabelecimentos de pequeno porte.

No ano de 2019, foi assinado um acordo de cooperação mútua entre o Idaf e os municípios com adesão ao Susaf/ES para realização das análises físicoquímicas e microbiológicas da água e de produto de origem animal dos estabelecimentos que aderiram ao Susaf/ES (ESPÍRITO SANTO, 2019a, b), o que contribuiu para realização das análises laboratoriais neste ano em específico.

Apenas o município de Cachoeiro de Itapemirim não foi contemplado com o convênio com o Idaf para a realização das análises laboratóriais, uma vez que o reconhecimento e adesão ao Sistema Unificado Estadual de Sanidade Agroindustrial Familiar de Pequeno Porte Susaf/ES ocorreu em novembro de 2019 (IDAF, 2020a), após a celebração do mesmo. Neste ano, o estabelecimento incluído ao Susaf/ES foi responsável pelo custeio das análises laboratoriais, com amostragem de queijo e iogurte (Tabela 2).

O município de Cariacica realizou uma maior amostragem de produtos nos estabelecimentos incluídos no Susaf/ES, coletando variedades diferentes de queijos nos estabelecimentos. A responsável pelo Serviço de Inspeção deste município afirmou ter realizado uma maior amostragem nos estabelecimentos devido à disponibilidade de verba para a realização das mesmas, verba esta proveniente do acordo de cooperação mútua com o Idaf.

O município de Linhares foi o único que realizou 2 análises no ano de 2019 para o mesmo estabelecimento, fato este informado pela equipe técnica do Serviço de Inspeção, por terem realizado uma análise previamente a indicação ao sistema Susaf/ES e outra prevista no programa de trabalho anual.

Os estabelecimentos são responsáveis pela qualidade dos alimentos que produzem. A realização das análises fiscais não desobriga o produtor de realizar as análises de autocontrole para monitoramento de seus processos produtivos e gerar confiabilidade da qualidade sanitária de seus produtos (BRASIL, 2017). De acordo com os Serviços de Inspeção Municipais consultados, os custos das análises laboratoriais estão entre as maiores dificuldades que os estabelecimentos enfrentam para a regulamentação das suas atividades e, por isso, não fazem parte da rotina dos pequenos estabelecimentos, como as agroindústrias familiares, a realização de análises de controle interno.

Apenas o município de Domingos Martins custea periodicamente parte das análises laboratoriais fiscais para os produtores registrados e os demais municípios ainda não possuem legislação que incluem o pagamento destas análises por parte do orçamento municipal, sendo as análises laboratoriais fiscais custeadas pelos próprios estabelecimentos produtores.

Vale ressaltar que há uma dificuldade dos pequenos produtores em compreender a necessidade e a importância da realização das análises laboratoriais dos 
produtos e, principalmente, de realizar o custeio das mesmas, por considerarem alto o custo de realização. Há ainda dificuldades em considerar os custos da realização das análises laboratoriais como parte dos custos de produção dos produtos, devendo os Serviços de Inspeção Municipais, juntamente com órgãos de extensão e outros setores que atuam no ramo, a contribuírem com o modelo sugerido para agroindústrias de pequeno porte, com a redução das exigências estruturais em decorrência da comprovação da qualidade sanitária do produto final por análises laboratoriais (BRASIL, 2018b).

Outro fato observado nos dados analisados diz respeito aos laboratórios de realização das análises no ano de 2019. Devido ao acordo de cooperação mútua entre Idaf e os municípios com a equivalência no Susaf/ES, a maior parte das análises foram realizadas em laboratório credenciado pela Rede Estadual de Laboratórios Agropecuários (Relagro), que possui sistema de qualidade implantado e acreditação pela ISO 17025 (Tabela 3).

O município de Cachoeiro de Itapemirim também realizou as análises em laboratório credenciado pela Rede Estadual de Laboratórios Agropecuários (Relagro), que possui sistema de qualidade implantado e acreditação pela ISO 17025 (Tabela 3).

Apenas os municípios de Santa Maria de Jetibá e Venda Nova do Imigrante realizaram análises laboratoriais de alguns dos seus estabelecimentos em um laboratório que não possuía credenciamento junto à Relagro (Laboratório 2) que também não apresentava acreditação no Instituto Nacional de Metrologia, Qualidade e Tecnologia (Inmetro) ou na ISO 17025, porém apresenta certificação ISO 9001. Esta norma estabelece requisitos para o sistema de gestão da qualidade de uma organização, com abordagem no desenvolvimento, implementação e melhoria da eficácia deste sistema, buscando oferecer um serviço de acordo com o especificado pelo cliente (ABNT NBR ISO 9001, 2015), porém não garante os requisitos necessários para garantia da qualidade de análises laboratóriais, que é o objetivo da norma ABNT NBR ISO 17025 (ABNT NBR ISO 17025, 2017).

A normal ISO 17025 é exclusiva para laboratórios de ensaio e calibração, garantindo a confiança na operação dos laboratórios que realizam as análises (ABNT NBR ISO 17025, 2017). Desta forma, a realização das análises fiscais em laboratórios credenciados à Relagro e acreditados pelo Inmetro e, que possuem a norma ISO 17025, garante aos clientes uma maior confiabilidade aos resultados, e respaldo legal para adoção das medidas previstas em casos de não conformidades, como apreensão de produtos e exigência de realização de novas análises laboratoriais para pesquisa de $L$. monocytogenes (BRASIL, 2009).

Apenas quatro laboratórios estão credenciados junto à Relagro, sendo que um deles é em Belo Horizonte e um em Juíz de fora (IDAF, 2020d). De acordo com os Serviços de Inspeção Municipais que realizaram análises no Laboratório 2, o fizeram pelo menor custo das análises, sendo que $o$ pequeno número de laboratórios credenciados no Estado pela Relagro é considerado um fator dificultador para a realização das análises.

Os resultados conformes nas análises laboratoriais para Listeria monocytogenes corroboram com a importância do estabelecimento estar registrado em um órgão fiscalizador, como o Serviço de Inspeção Municipal, que tem como função orientar e capacitar os produtores em temas relativos a qualidade do leite e a aplicação das boas práticas de fabricação, e também verificar continuamente nas fiscalizações as condições higiênicas e de organização das agroindústrias, como controle da matériaprima, dos lotes de produtos, da higiene 
das câmaras frigoríficas e outras medidas para evitar a contaminação microbiana e contribuir para a qualidade dos derivados lácteos elaborados.

Todos os estabelecimentos realizaram as análises laboratoriais no ano de 2019, porém podemos inferir que o fator facilitador foi o custeio pelo Idaf, pois a maior parte dos municípios realizou uma única análise neste ano, e de apenas um produto. Devemos ressaltar ainda a importância do programa de equivalência Susaf/ES para o fortalecimento das agroindústrias de pequeno porte e maior segurança alimentar para o consumidor, pois a exigência da realização das análises laboratoriais contribui com a qualidade dos produtos produzidos no estado do Espírito Santo.

Apesar dos esforços conjuntos e da criação do programa Susaf/ES, observouse que há uma desuniformidade na amostragem dos produtos lácteos para realização das análises laboratoriais. Sugere-se que o órgão que faz o reconhecimento da equivalência do Susaf/ES estabeleça diretrizes e parâmetros para orientar os municípios a respeito da padronização da amostragem dos produtos a serem analisados.

Por fim, políticas governamentais devem fomentar a realização das análises laboratoriais em agroindústrias de pequeno porte, compartilhando os custos das análises entre poder público e responsáveis legais dos empreendimentos, contribuindo assim para maior frequência de realização das análises e maior amostragem dos produtos elaborados. Desta forma, será possível a sustentabilidade do agronegócio, permanência das famílias no campo e oferta de produtos com maior qualidade sanitária aos consumidores.

\section{CONCLUSÕES}

Os resultados conformes nas análises laboratoriais demonstram a importância das indústrias produtoras de laticínios estarem registradas nos órgãos fiscalizadores, como o Serviço de Inspeção Municipal, que certificam a qualidade e a segurança dos alimentos produzidos nestes estabelecimentos.

Apesar de todos os estabelecimentos terem realizado análises laboratoriais no período avaliado, a amostragem de um único produto de apenas um lote, e uma única variedade, podem ser fatores limitantes para afirmação da ausência de Listeria monocytogenes, tendo em vista os diferentes parâmetros intrínsecos e extrínsecos dos alimentos que podem afetar na viabilidade do patógeno.

\section{AGRADECIMENTOS}

Aos Serviços de Inspeção Municipais dos municípios aderidos ao Sistema Unificado Estadual de Sanidade Agroindustrial Familiar de Pequeno Porte - Susaf/ES, participantes deste estudo; à equipe da Gerência de Agroindústria de Pequeno Porte do Idaf e ao curso de Pós-graduação em Controle de Qualidade e Segurança de Alimentos do Instituto Federal do Espírito Santo - Ifes - Campus Piúma.

\section{REFERÊNCIAS}

ABNT NBR ISO 9001, 2015. Gestão da
qualidade - Sistemas de gestão da qualidade - Requisitos. Disponível em: $<$ https://fatorsyn.com.br/iso-9001-2015norma-completa-em-pdf-download/>. Acesso em: 02 de ago. de 2020.
ABNT NBR ISO 17025, 2017. Requisitos gerais para a competência de laboratórios de ensaios de calibração. Disponível em:
$<$ https://www.normas.com.br/visualizar/ab nt-nbr-nm/20966/abnt-nbriso-iec17025- requisitos-gerais-para-a-competencia-de- laboratorios-de-ensaio-e-calibracao>. Acesso em: Acesso em: 02 de ago. de 2020. 
BARANCELLI, G. V.; SILVA-CRUZ, J. V.; PORTO, E.; OLIVEIRA, C. A. F. Listeria monocytogenes: Ocorrência em produtos lácteos e suas implicações em saúde pública. Arquivos do Instituto Biológico, v. 78, n. 1, p. 155-168, 2011.

BRASIL. MINISTÉRIO DA AGRICULTURA PECUÁRIA E ABASTECIMENTO. Regulamento técnico de identidade e qualidadede queijos. Portaria n. 146 de 07 de março de 1996. Brasília, 1996. Disponível em: http://sistemasweb.agricultura.gov.br/sisleg is/action/detalhaAto.do?method=gravarAto PDF\&tipo $=$ POR \&numeroAto $=00000146 \&$ seqAto $=000 \&$ valorAno $=1996 \&$ orgao $=\mathrm{MA}$ RA\&codTipo $=\&$ desItem $=\&$ desItemFim $=$. Acesso em: 4 abr. 2020.

BRASIL. MINISTÉRIO DA AGRICULTURA PECUÁRIA E ABASTECIMENTO. Procedimentos de Controle da Listeria monocytogenes em produtos de origem animal prontos para o consumo. Brasília, 2009. Disponível em: https://www.gov.br/agricultura/assuntos/in specao/produtos-animal/controle-depatogenos/arquivos-controle-depatogenos/in_09-

_de_8_de_abril_de_2009.pdf. Acesso em: 4 abr. 2020.

BRASIL. MINISTÉRIO DA

AGRICULTURA PECUÁRIA E ABASTECIMENTO. Decreto $\mathbf{n}^{\circ} \mathbf{9 0 1 3}$ de $\mathbf{2 9 / 0 3 / 2 0 1 7}$. Regulamenta as leis $1283 / 1950$ e 7889/1898, que dispõem sobre a inspeção industrial e sanitária de produtos de origem animal. Brasília, 2017. Disponível em:<

http://www.planalto.gov.br/ccivil_03/_ato2 015-2018/2017/decreto/d9013.htm>.

Acesso em: 2 de ago. de 2020.

BRASIL. 2018a. MINISTÉRIO DA AGRICULTURA PECUÁRIA E ABASTECIMENTO. INSTRUÇÃO NORMATIVA N ${ }^{\circ} 76$, DE 26 DE
NOVEMBRO DE 2018. Aprova os

Regulamentos Técnicos que fixam a identidade e as características de qualidade que devem apresentar o leite cru refrigerado, o leite pasteurizado e o leite pasteurizado tipo A. Diário Oficial da União, Brasília, 2018. Disponível em: < http://www.in.gov.br/materia/-

/asset_publisher/Kujrw0TZC2Mb/content/i d/52750137/do1-2018-11-30-instrucaonormativa-n-76-de-26-de-novembro-de2018-52749894IN\%2076> Acesso em: 21 de nov. de 2019.

BRASIL, Carolina. 2018b. Idaf cria selo estadual menos burocrático para agroindústrias de pequeno porte. Folha online, 15 de junho de 2018. Disponível em: https://www.folhaonline.es/idaf-cria-seloestadual-menos-burocratico-paraagroindustrias-de-pequeno-porte/. Acesso em: 11 de jul. de 2020.

BRASIL, ANVISA - Agencia Nacional De Vigilância Sanitária. INSTRUÇÃO NORMATIVA N ${ }^{\circ} 60$, DE 23 DE DEZEMBRO DE 2019a. Disponível em: http://www.in.gov.br/en/web/dou//instrucao-normativa-n-60-de-23-dedezembro-de-2019-235332356. Acesso em: 15 de maio de 2020.

BRASIL, Ministério da Agricultura Pecuária e Abastecimento, 2019b. Lista de Parâmetros Físico-Químicos e Microbiológicos para Produtos de Origem Animal Comestíveis e Água de Abastecimento. Disponível em: < https://www.gov.br/agricultura/ptbr/assuntos/inspecao/produtosanimal/analises-laboratoriais> . Acesso em: 11 set. de 2020.

CALLON, Cécile; PICQUE, Daniel; CORRIEU, Georges; MONTEL, MarieChristine. Ripening conditions: A tool for the control of Listeria monocytogenes in uncooked pressed type cheese. Food Control, v. 22, n. 12, p. 1911-1919, 2011. DOI: 
https://doi.org/10.1016/j.foodcont.2011.05. 003.

CAMARGO, Anderson Carlos; WOODWARD, Joshua John; CALL, Douglas Ruben; NERO. Listeria monocytogenes in Food-Processing Facilities, Food Contamination, and Human Listeriosis: The Brazilian Scenario. Foodborne pathogens and disease, v. 14, n.11, 2017. DOI:

https://doi.org/10.1089/fpd.2016.2274

CHOI, Min Hyuk; PARK, Yu Jin; KIM, Myungsook; SEO,Young Hee; KIM, Young Ah; CHOI, Jun Yong; YONG, Dongeun; JEONG, Seok Hoon; LEE, Kyungwon. Increasing incidence of listeriosis and infection associated clinical outcomes. Annals of Laboratory Medicine, v.38, p. 102-109, 2018. DOI: https://doi.org/10.3343/alm.2018.38.2.102

DIAS, Maria Angélica Costa; SANT'ANA, Anderson S.; CRUZ, Adriano G.; FARIA, José de Assis F.; OLIVEIRA, Carlos Augusto Fernandes; BONA, Evandro

On the implementation of good manufacturing practices in a small processing unity of mozzarella cheese in Brazil. Food Control, n. 24, p. 199-205, 2012. DOI:

https://doi.org/10.1016/j.foodcont.2011.09. 028.

D’AMICO, Dennis J.; DRUART, Marc J. ; DONNELLY, Catherine W.

W. 60-day aging requirement does not ensure safety of surface-mold-ripened soft cheeses manufactured from raw or pasteurized milk when Listeria monocytogenes is introduced as a postprocessing contaminant. Journal of food protection, v. 71 , n. 8 , p. 1563-1571, 2008.

DI CICCIO, Pierluigi; RUBIOLA; Selene; GRASSI, Maria Ausilia; CIVERA, Tiziana; ABBATE, Francesco; CHIESA,
Francesco. Fate of Listeria monocytogenes in the Presence of Resident Cheese Microbiota on Common Packaging Materials. Frontiers in Microbiology, v. 11, 2020. DOI: https://doi.org/10.3389/fmicb.2020.00830.

DONNELLY, Catherine W. Growth and Survival of Microbial Pathogens in Cheese. Cheese: Chemistry, Physics and Microbiology, v. 1, p. 541-559, 2004.

ESPÍRITO SANTO. Assembleia Legislativa do Estado do Espírito Santo. Instrução Normativa $\mathbf{N}^{0} 010$ de 16/09/2014. Institui o Regime especial de fiscalização. 2014. Disponível em: https://idaf.es.gov.br. Acesso em: 4 abr. 2020.

ESPÍRITO SANTO. Assembleia Legislativa do Estado do Espírito Santo. Decreto $n^{0}$ 4308-R, de 21 de setembro de 2018. Atualiza os procedimentos e requisitos necessários para adesão dos Municípios ao Sistema Unificado Estadual de Sanidade agroindustrial de produtos de origem animal no estado do Espírito Santo. Vitória - ES, 2018. Disponível em: https://idaf.es.gov.br/base-legal-do-susafes. Acesso em: 4 abr. 2020.

\section{ESPÍRITO SANTO. Diário oficial do} Espírito Santo de13 de setembro de 2019a. Acordo de cooperação mútua ACM No 0007/2019 a 0011/2019.

Realização de análises laboratoriais em estabelecimentos incluídos no Sistema Unificado Estadual de Sanidade Agroindustrial Familiar de Pequeno Porte (Susaf/ES). Disponível em:< http://ioes.dio.es.gov.br/portal/visualizacoe s/diario_oficial>. Acesso em: 05 de julho de 2020.

\section{ESPÍRITO SANTO. Diário oficial do} Espírito Santo de 18 de setembro de 2019b. Acordo de cooperação mútua ACM No 006/2019. Realização de análises laboratoriais em estabelecimentos 
incluídos no Sistema Unificado Estadual de Sanidade Agroindustrial Familiar de Pequeno Porte (Susaf/ES). Disponível em: $<$

http://ioes.dio.es.gov.br/portal/visualizacoe s/diario_oficial>. Acesso em: 05 de julho de 2020.

GAMEIRO, Noémia; FERREIRA-DIAS, Suzana; FERREIRA, Mass; BRITO, Luisa. Evolution of Listeria monocytogenes populations during the ripening of naturally contaminated raw ewe's milk cheese. Food Control, v. 18, n. 10, p. 1258-1262, 2007. DOI:

https://doi.org/10.1016/j.foodcont.2006.08. 002.

GANZ, Kyle; YAMAMOTO, Etsuko; HARDIE, Kate; HUM, Christine; HUSSEIN, Hussein; LOCAS, Annie; STEELE, Marina. Microbial safety of cheese in Canada. International Journal of Food Microbiology, v. 321, 2020. DOI: https://doi.org/10.1016/j.ijfoodmicro.2020. 108521

GÉRARD, Amaury; EL-HAJJAJI, Soundous; NIYONZIMA, Eugène; DAUBE, Georges; SINDIC, Marianne. Prevalence and survival of Listeria monocytogenes in various types of cheese-A review. International Journal of Dairy Technology, v. 70, 2018. DOI: https://doi.org/10.1111/1471-0307.12552

GIACOMETTI, Federica; DAMINELLI, Paolo; FIORENTINI, Laura; COSCIANICUNICO, Elena; MONASTERO, Paola; DALZINI, Elena; LOSIO, Marina-Nadia; DELL'ORFANO, Giovanni; ROSSINI, Rachele; PIVA, Silvia; SERRAINO, Andrea. Behavior of Listeria innocua during the manufacturing and pit-ripening of Formaggio di Fossa di Sogliano PDO cheese. Italian Journal of Food Safety, v.9, n.8552, p. 114- 119, 2020.

HENTGES, Denise; SILVA, Daiani Teixeira; DIAS, Priscila Alves;
CONCEIÇÃO, Rita de Cássia dos Santos; ZONTA, Miriam Nunes; TIMM, Cláudio Dias. Pathogenic microorganism survival in dulce de leche. Food Control, v.21, p. 1291-1293, 2010. DOI:

https://doi.org/10.1016/j.foodcont.2010.02. 014

HUNT, Karen; DRUMMOND, Niall; MURPHY, Mary; BUTLER, Francis; BUCKLEY, Jim; JORDAN, Kieran. A case of bovine raw milk contamination with Listeria monocytogenes. Irish Veterinary Journal, v. 65, p. 13, 2012. DOI: https://doi.org/10.1186/2046-048165-13.

IDAF - Instituto de Defesa Agropecuária e Florestal do Espírito Santo. Sistema Unificado Estadual de Sanidade Agroindustrial Familiar de Pequeno Porte (Susaf/ES). 2020a. Disponível em: https://idaf.es.gov.br/susaf. Acesso em: 4 abr. 2020.

IDAF - Instituto de Defesa Agropecuária e Florestal do Espírito Santo. 2020b.

Consulta pública número 001/2020. Minuta: análise laboratorial fiscal de produtos e institui o Regime Especial de Fiscalização - REF. Disponível em: $<$ https://idaf.es.gov.br/consultas-publicasidaf $>$. Acesso em: 20 de abr. de 2020.

IDAF - Instituto de Defesa Agropecuária e Florestal do Espírito Santo. 2020c Nota técnica 001/2020. Estabelece a periodicidade de coleta de amostras e os parâmetros para análise fiscal em estabelecimentos registrados junto ao Serviço de Inspeção Agroindustrial de Pequeno Porte (Siapp). Disponível em: $<$ https://idaf.es.gov.br/Media/idaf/Acesso \%20r\%C3\%A1pido/9.\%20Agroind\%C3\% BAstria/Siapp/Nota\%20tecnica\%200012020\%20Periodicidade\%20de\%20coleta\% 20e\%20para\%C2\%A2metros\%20para\%20 analise $\% 20$ fiscal\%20de\%20agua\%20e\%20 de\%20produto.pdf $>$. Acesso em: 25 de out de 2020. 
IDAF - Instituto de Defesa Agropecuária e Florestal do Espírito Santo. Lista dos laboratórios credenciados na Relagro, 2020d. Disponível em:

$<$ https://idaf.es.gov.br/Media/idaf/Acesso \%20r\%C3\%A1pido/5.\%20Diagn\%C3\%B3 stico\%20laboratorial/Lista\%20de\%20Labo rat\%C3\%B3rios\%20Credenciados\%20\%20RELAGRO\%20-\%20Rev.001\%20$\% 2004.10 .18$.pdf $>$ Acesso em: 04 de out. de 2020.

KASALICA, A.; VUKOVIC, V.; VRANJES, A.; MEMISI, N. Listeria monocytogenes in milk and dairy products. Biotechnology in Animal Husbandry, v. 27, n. 3, p. 1067-1082, 2011. DOI: https://doi.org/10.2298/BAH1103067K.

LEE, Jeeyeon; SEO, Yeongeun; HÁ, Jimyeong; KIM, Sejeong; CHOI, Yukyung; OH, Hyemin; Lee, YEWON; KIM, Yujin; KANG, Joohyun; PARK, Eunyoung; YOON, Yohan. Influence of milk microbiota on Listeria monocytogenes survival during cheese ripening. Food Science \& Nutrition, v. 00, p.1-6, 2020. DOI: https://doi.org/10.1002/fsn3.1806.

OLIVEIRA, Naila Albertina; BITTENCOURT, Gabriela Marques; BARANCELLI, Giovana Verginia; KAMIMURA, Eliana Setsuko; IN LEE, Sarah Hwa; OLIVEIRA, Carlos Augusto Fernandes. Listeria monocytogenes in Brazilian foods: occurrence, risks to human health and their prevention.

Current Research Nutrition and Food Science, v. 7, n.2, p. 320-330, 2019. DOI: http://dx.doi.org/10.12944/CRNFSJ.7.2.02

PAPIĆ, B.; GOLOB, M.; KUŠAR, D.; PATE, M.; ZDOVC, I. Source tracking on a dairy farm reveals a high occurrence of subclinical mastitis due to hypervirulent Listeria monocytogenes clonal complexes. Journal of Applied Microbiology, v. 127, n.5, p.
1349 -1361, 2019 DOI:

https://doi.org/10.1111/jam.14418.

PANT, R. R.; PRAKASH, G.;

FAROOQUIE, J. A. A framework for traceability and transparency in the dairy supply chain networks. Procedia - Social and Behavioral Sciences, n. 189, p. 385 394, 2015. DOI:

https://doi.org/10.1016/j.sbspro.2015.03.23 5.

SKOWRON, Krzysztof; HULISZ, Karolina; GRYŃ, Grzegorz; OLSZEWSKA, Halina; WIKTORCZYK, Natalia; PALUSZAK, Zbigniew. Comparison of selected disinfectants efficiency against Listeria monocytogenes biofilm formed on various surfaces. International Microbiology, v. 21, p. 23-33, 2018. DOI: https://doi.org/10.1007/s10123-018-0002-5

SZCZAWIŃSKI, J.; SZCZAWIŃSKA, M. E.; ŁOBACZ, A.; JACKOWSKA-TRACZ, A. Modeling the effect of temperature on survival rate of Listeria monocytogenes in yogurt. Polish Journal of Veterinary Sciences, v. 19, n. 2, p.317-324, 2016. DOI: http://dx.doi.org/10.1515/pjvs-20160039

TIRLONI, E.; BERNARDI, C.; ROSSHAUG, P. S.; STELLA, S. Potential growth of Listeria monocytogenes in Italian mozzarella cheese as affected by microbiological and chemical-physical environmen. Journal of Dairy Science, v. 102, p. 4913-4924, 2019. DOI: https://doi.org/10.3168/jds.2018-15991

THOUVENOT, Pierre; VALES, Guillaume; BRACQ-DIEYE, Hélène; TESSAUD-RITA, Nathalie, MAURY, Mylène M.; MOURA, Alexandra; LECUIT, Marc; LECLERCQ, Alexandre. MALDI-TOF mass spectrometry-based identification of Listeria species in surveillance: A prospective study. Journal 
of Microbiological Methods, v. 144, p. 29-32, 2018.

THOMAS, Merlyn; TIWARI, Ratnesh; MISHRA, Abhinav. Predictive model of Listeria monocytogenes growth in queso fresco. Journal of Food Protection, v. 82, N. 12, p. 2071-2079, 2019. DOI: https://doi.org/10.4315/0362-028X.JFP-19185.

VALLIM, Deyse Christina; HOFER, Cristina Barroso; LISBÔA, Rodrigo de Castro; BARBOSA, André Victor; RUSAK, Leonardo Alves; REIS, Cristhiane Moura Falavina; HOFER, Ernesto. Twenty Years of Listeria in Brazil: Occurrence of Listeria Species and Listeria monocytogenes Serovars in Food Samples in Brazil between 1990 and 2012. BioMed Research International, v. 2015, p. 1- 8, 2012. DOI:

http://dx.doi.org/10.1155/2015/540204

VINHA, Mariana Barboza; DIAS, Rachel Quandt. Diagnóstico da agroindústria familiar no Espírito Santo: Resultado da pesquisa 2018. Vitória - ES: Instituto Capixaba de Pesquisa, Assistência Técnica e Extensão Rural (Incaper), 2019.

WEMMENHOVE, E.; WELLS-BENNIK, M. H. J.; STARA, A.; VAN HOOIJDONK, A. C. M.; ZWIETERING, M. H. How $\mathrm{NaCl}$ and water content determine water activity during ripening of Gouda cheese, and the predicted effect on inhibition of Listeria monocytogenes.

Journal of Dairy Science, v. 99, p.1-10, 2016. DOI:

http://dx.doi.org/10.3168/jds.2015-10523 